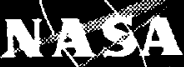

\title{
Models for Liquid Impact Onboard Sloshsat FLEVO
}

Jan P.B. Vreeburg

National Aerospace Laboratory NLR, Amsterdam, The Netherlands

David J. Chato

Glenn Research Center, Cleveland, Ohio

Prepared for the

Space 2000 Conference and Exposition

sponsored by the American Institute of Aeronautics and Astronautics

Long Beach, California, September 19-21, 2000

National Aeronautics and

Space Administration

Glenn Research Center 


\section{Acknowledgments}

Sloshsat FLEVO is a harmonized programme between the European Space Agency (ESA) and The Netherlands Agency for Aerospace Programs (NIVR). Main contractor is the National Aerospace Laboratory NLR (The Netherlands) with participation of Fokker Space (The Netherlands), Verhaert (Belgium), Rafael (Israel) and NASA (USA). The Sloshsat FLEVO development is performed in the framework of the ESA Technology Development Programme (TDP) Phase 2 and the NIVR Research and Technology (NRT) programme.

Available from

NASA Center for Aerospace Information 7121 Standard Drive

Hanover, MD 21076

Price Code: A03
National Technical Information Service 5285 Port Royal Road Springfield, VA 22100 Price Code: A03

Available electronically at http://gltrs.grc.nasa.gov/GLTRS 


\title{
MODELS FOR LIQUID IMPACT ONBOARD SLOSHSAT FLEVO
}

\author{
Jan P.B. Vreeburg \\ National Aerospace Laboratory NLR \\ Amsterdam, The Netherlands \\ David J. Chato \\ National Aeronautics and Space Administration \\ Glenn Research Center \\ Cleveland, Ohio 44135
}

\begin{abstract}
Orbital experiments on the behavior of liquid in spacecraft are planned. The Sloshsat free-flyer is described. Preparation of the experiments, and later evaluation, are supported by models of varying complexity. The characteristics of the models are discussed. Particular attention is given to the momentum transfer between the liquid and the spacecraft, in connection with the liquid impact that may occur at the end of a reorientation maneuver of the spacecraft.
\end{abstract}

\section{INTRODUCTION}

The European Space Agency (ESA) in conjunction with the Netherlands Agency for Aerospace Programs has undertaken a project to investigate fluid motion in zero-g. ESA has contracted with the Netherlands National Aerospace Laboratory (Nationaal Lucht-en Ruimtevaartlaboratorium or NLR) for a small free flying spacecraft deployed from the shuttle but flying in tandem with it. This spacecraft is known as the Sloshsat Facility for Liquid Experimentation and Verification On-orbit (Sloshsat FLEVO or just Sloshsat for short). 'NASA participates in the program and provides the flight opportunity. The planned investigations are detailed in an Experiment Definition Document. A general objective for all experiments is to validate their Computational Fluid Dynamics (CFD) models, but NASA has the particular goal to demonstrate that propellant can be settled at low $(\mathrm{Bo}<12)$ thrust levels, ${ }^{2}$ either continuously or achieved by on/off modulation. An objective of NLR is to validate its Sloshsat Motion Simulator (SMS) ${ }^{3}$ for use in control algorithms of spacecraft.

\section{NOMENCLATURE}

Figure 3 entries:

$\mathrm{m}=$ slug mass

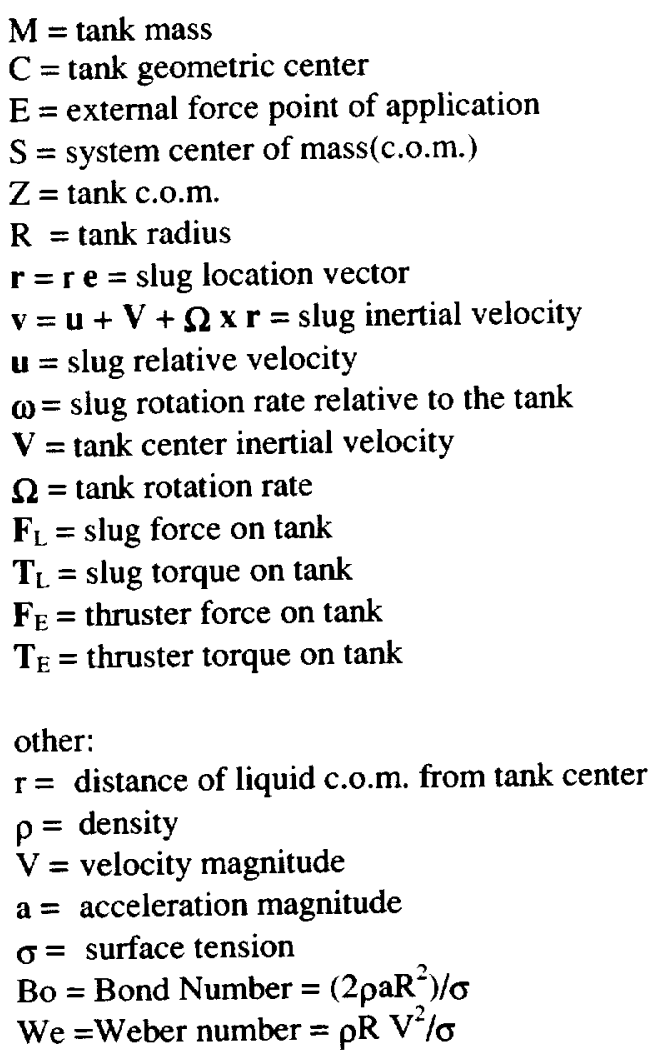

\section{BACKGROUND}

If a vehicle contains a partially filled tank with liquid, problems may arise from the unpredictability of the liquid reaction forces in response to a steering maneuver. A recent example from space is supplied by the NEAR spacecraft that automatically interrupted its insertion burn when fuel reaction was larger than anticipated. This prevented NEAR from orbiting its target asteroid Eros and delayed the mission almost a year (The next try was successful in putting NEAR in its orbit about asteroid Eros ${ }^{4}$ ). Another problem, and one that occurs even for negligible magnitude of the liquid mass fraction, comes from the liquid distribution in a partially filled 
tank. Most liquid fueled rocket engines require a continuous supply of liquid propellant to function. Low gravity challenges these engines since the lack of separation between liquid and gas will allow gas ingestion into an engine, possibly resulting in a catastrophic failure of that engine. However, coasting in the low gravity environment for long periods of time (1/2 hour to days) is required by most upper stage rockets to correctly position their payloads. Prior to restarting the engines of these rockets, something must be done to assure gas free liquid to the engines. Satisfactory solutions to liquid slosh and fluid management problems would contribute to the introduction of high-performance service vehicles about ISS.

\section{DESCRIPTION OF SPACECRAET}

Sloshsat FLEVO is a free flying spacecraft launched from a cross-bay Hitchhiker bridge attached to the Space Shuttle. The primary test-bed is an 87-liter composite tank filled with 33.5 liters of deionized water. Platinum wire rings embedded in the tank wall measure capacitance at 270 locations throughout the tank. This information is used to determine liquid depth over the location to a maximum resolution of $0.03 \mathrm{~m}$. Miniature thermal dispersion meters provide velocity measurements at 10 locations in the tank. A cold gas thruster system fed by four gaseous nitrogen tanks allows the tank to be maneuvered through a broad range of motions. Twelve $0.8 \mathrm{~N}$ thrusters provide the ability to translate in all three directions and rotate about all three axes. Six accelerometers and 3 gyroscopes provide detailed information on Sloshsat motion. An on-board telemetry system relays experiment data back to the Space Shuttle for transmission to the ground. Figure 1 shows the interior of the Sloshsat.

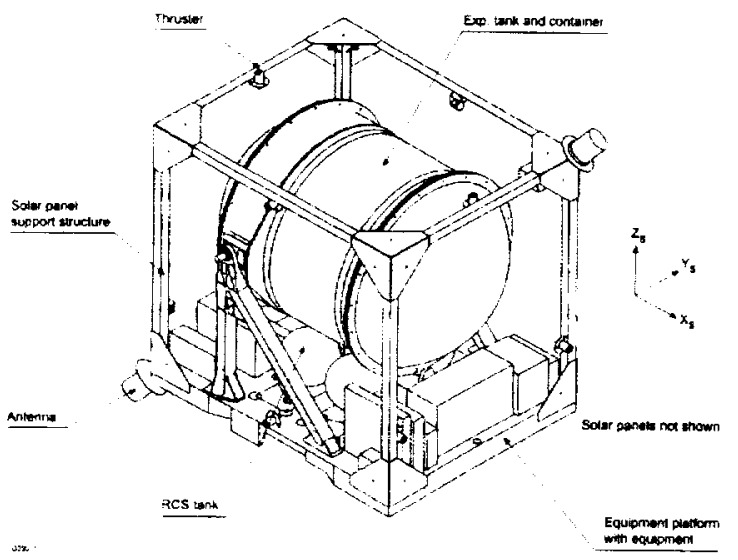

Figure 1 Sloshsat Interior

\section{SYSTEM MODELING}

The investigators with Sloshsat experiments use different models according to their objectives. For spacecraft dynamics the model must provide reliable predictions of the force and torque profile during maneuvers. This also implies that the prediction of the liquid center of mass (c.o.m.) should be accurate. Since the model is often part of a mission design tool it must be simple and CFD codes are generally considered not suitable except for final verification use. For liquid management investigations the emphasis is on good prediction of the liquid shape and, again, liquid c.o.m. location. These goals can be realized only via CFD.

The art of model development is disclosed in the selection of type and number of variables. The number of variables corresponds to the degrees of freedom of the model and one generally strives to minimize the number. A model needs to be checked for correctness, called verification, which is accomplished by monitoring conserved quantities (momentum, energy) as predicted during operation of the model and by recalculation of worked problems from the literature. The test of model performance, called validation, is realized by comparing its predictions with the outcome of physical experiments. In some cases comparison with more sophisticated or already validated models counts as validation.

\section{IUSTIEICATION}

Experiments with Sloshsat are deemed necessary for the validation of models because the outcome of these experiments cannot be predicted with confidence. A successful mission is to provide a comprehensive set of data to benefit many model developers.

Previous investigations have indicated that the cause of failure to predict may be with two phenomena:

- the motion of the liquid-solid contact line

- the damping of the liquid motion.

\section{SLOSHSAT MODELS}

At present four models are used for Sloshsat mission preparation. Two are for spacecraft dynamics analysis and use lumped mass liquid, and two are CFD programs. The models have been developed independently and their predictions are compared for verification and for the assessment of range of application. In keeping with the Sloshsat mission 
characteristics the models are meant for large amplitude, low Bond number liquid motion. Much of the earlier literature covers small amplitude slosh. ${ }^{5.6}$ Illustrations of results from the four models are here limited to the liquid transfer, or reorientation, maneuver. However, many rotational (stability) results have been generated also, for preparation of Sloshsat control.

\section{SMS}

The Sloshsat Motion Simulator ${ }^{3,10}$ is the first model specifically developed for the Sloshsat mission. Its architecture is depicted in figure 2; the annotations are defined in the Nomenclature. The tank principal inertia tensor is also tracked by the model. SMS is coded in Fortran and is operated in the EuroSim environment. It predicts the 3-D motions of the tank and of the liquid c.o.m. The liquid c.o.m. is not constrained to a surface but can be anywhere in a spherical volume of the cavity of radius $R$. This freedom is a consequence of the liquid model: a sphere of constant mass but variable radius, named 'slug'. The slug has variable moment of inertia and internal energy from capillary (surface) potential and from kinetics. Its resonance frequencies are also variable and have values between those of liquid drops and bubbles of slug size.

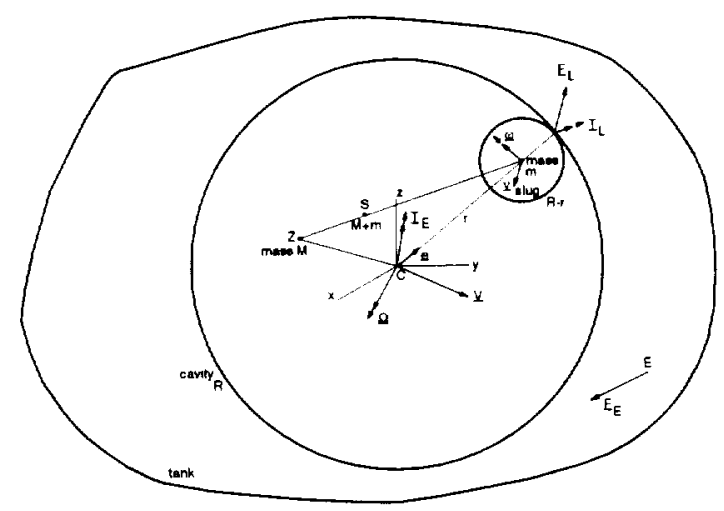

Figure 2 Architecture of SMS

\section{ComElo}

The ComFlo CFD code has been under development since $1995 .^{9}$ Comparable to the well-known FLOW3D code, it is based on the Volume of Fluid (VOF) method, but it has important advantages when used for Sloshsat performance prediction. The code is capable of stable simulation of the coupled liquidsolid dynamics for any value of the liquid mass fraction. Being developed specifically for low Bond number flows, it provides accurate tracking of the free surface shape and curvature, and without problem starts from or terminates to hydrostatic equilibrium configurations. The latter feature is often mentioned as lacking in FLOW-3D, due to its development as a gravity slosh method.

Comparison of ComFlo and SMS predictions of interaction force and of liquid c.o.m. trajectory showed satisfactory agreement.

\section{ECIIPSE}

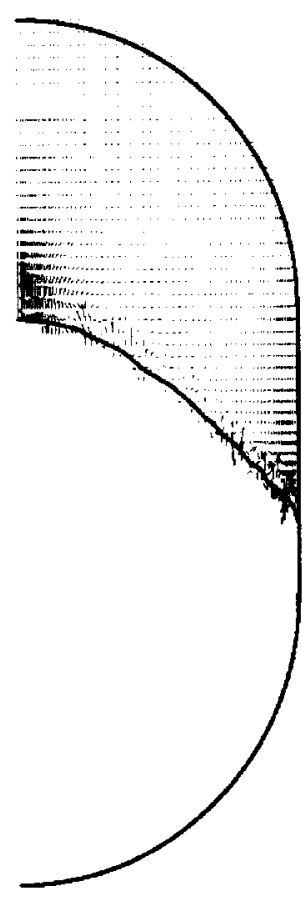

a) 5 seconds

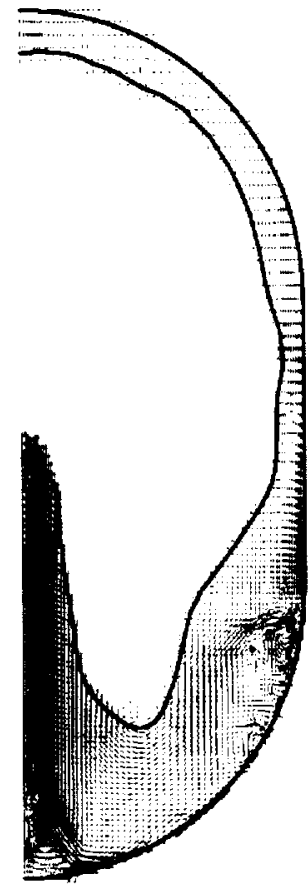

b) 33.6 seconds
Two Sloshsat Thrusters Firing Together for a 0.33 second pulse every second (Effective Bo $=4$ )

Figure 3 ECLIPSE simulation result

The ECLIPSE code has a long history of use with reorientation problems. ${ }^{7}$ ECLIPSE solves the NavierStokes equation in a radially symmetric formulation using a VOF approach. A color function is used to track the free surface position. Free surface forces are approximated in this version of ECLIPSE by the method of Kothe. ${ }^{8}$ An illustration of a prediction of a transfer maneuver is in figure 3 . Heavy black lines show the tank wall and free surface location(s). Fine lines show vectors of velocity. Since in each image the vectors are scaled from the maximum in that 
image, they should be viewed for qualitative rather than quantitative information. ECLIPSE simulations of the Sloshsat settling maneuvers can be found in Chato.'

\section{Allips2}

This is a Matlab model of a two-dimensional rigid body (the tank) in contact with a movable point mass (the liquid) that is constrained to an (approximate) elliptical trajectory. Such a model was developed for use in the STARDUST mission ${ }^{12}$ but allips 2 has an additional interaction. It is force $S$ that acts normal to the direction towards the center of the ellipse, to model the capillary action for a minimum energy configuration in the tank. Such configuration has all liquid at one pole of the tank.

The constitutive equation for $S$ has two parameters, sf and sp, as follows:

$S=\operatorname{sf} \varphi^{\prime}+s p \sin 2 \varphi / r$

where $\varphi=$ the angular coordinate of the point mass on its trajectory, and $\varphi^{\prime}$ its rate of change, or 'swirl'. Parameter sf controls the viscous friction; sp models the capillary potential effect.

Predictions by allips 2 have been compared with similar predictions generated by the 3-D model, SMS. For the comparison the liquid point mass trajectory was set to be a circle, and SMS was extended to also provide a capillary potential as governed by sp. Figure 4 illustrates the different predictions for the velocity components of the liquid c.o.m. during a reorientation maneuver of Sloshsat. The differences can be explained, and comparisons of other dynamic variables show similarly good agreement, which serves to verify either model.

Both allips2 and SMS have shown that the liquid transfer duration depends strongly on the control gain and on the delay time between the tank rate measurement and the instance of correction torque activation. The 'elasticity' of the slug in SMS has a significant effect on the initial transfer of momentum between the slug and the tank.

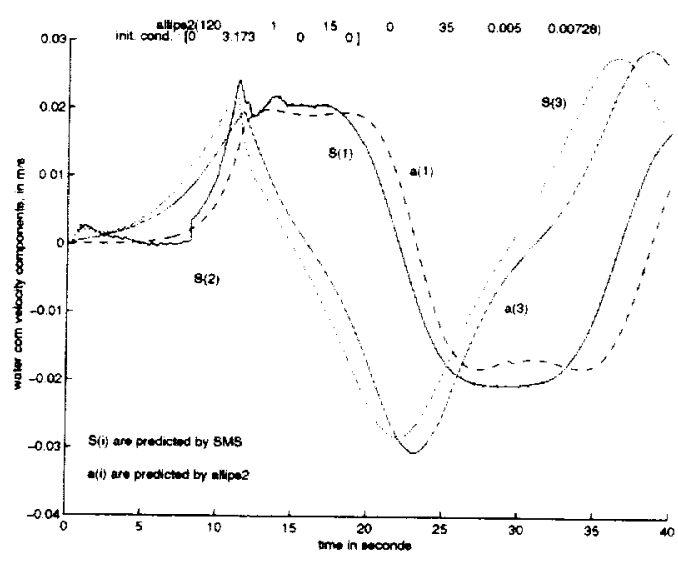

Figure 4 Cartesian components of the liquid c.o.m. velocity in a spherical/circular cavity

As a performance test the experimental results of Masica ${ }^{13}$ have been investigated with the model. For liquid in a cylindrical tank, Masica determined at what constant acceleration normal to the tank centerline the liquid free surface becomes unstable and starts to flow. He found that for water (high surface tension) the critical Bond number is about 2.5 . Liquid with low surface tension gave a value 1.12 and one may suspect some effect of stiction at the contact line for the water value.

The value of the thrust for this run was reduced to $0.194 \mathrm{~N}$, capable to accelerate the system at $0.194 /(95.3+33.5)=0.0015 \mathrm{~m} / \mathrm{s}^{2}$. Then the Bond number, for water, becomes:

$\mathrm{Bo}=\rho \mathrm{aR}^{2} / \sigma=1000 * 0.0015 * 0.228^{2} / 0.07=1.12$

At this, the low value of Masica, the water motion should be stable and not break from its initial side of the tank, even if stiction effects are not operative. By trial and error was found that the stable condition requires a magnitude of 0.0057 for $\mathrm{sp}$, value 0.0056 is unstable. Figure 5 shows the motion of the liquid point mass for these values.

The capillary force parameter $s p=2 \sigma R^{2}$ has value 0.00728 for the Sloshsat data. This means that the critical thrust level is predicted to be $0.25 \mathrm{~N}$, or the critical Bond number at the reasonable value 1.46 . 


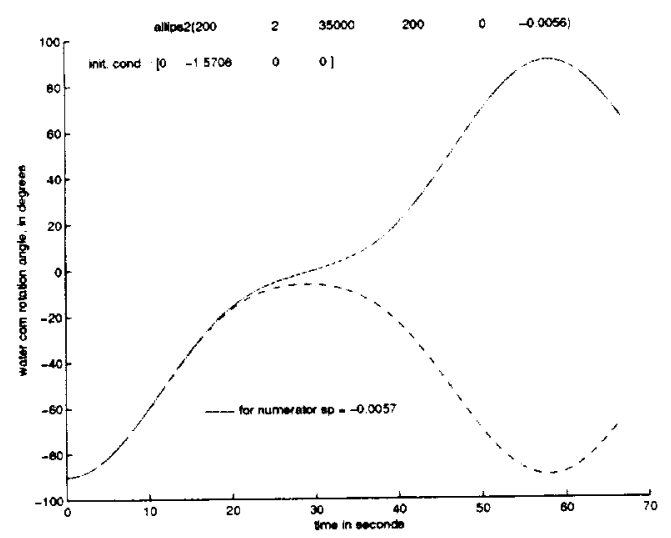

Figure 5. Liquid c.o.m. angular locations, from the minor axis of the elliptical trajectory

The maneuver that generated figure 3 is predicted by allips 2 as recorded in figure 6 . Two curves are shown that correspond to slightly different initial conditions for the liquid point mass and a consequently different transfer trajectory. The dashed line transfer time is comparable to the value found by the CFD simulation but the solid line transfer takes considerably longer. The longer duration transfer (solid line) has the masspoint passing far from the tank c.o.m. As in Sloshsat, the tank c.o.m. is not at the geometrical center of its cavity; it is offset from the major axis of the trajectory ellipse in allips 2 .



Figure 6 Transfer predictions by allips 2
Also of interest to the Sloshsat team are the forces exerted by the fluid motion and the commanded control thruster firings. Allips 2 predictions of these quantities, for the transfers of figure 6 , are shown in figures 7 and 8 . It is to be noted that the control torque activations are reflected in the peaks of the liquid-tank interaction forces.

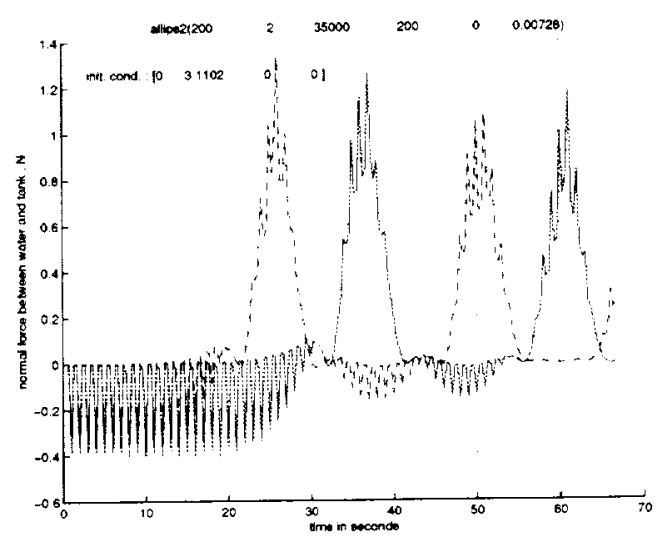

Figure 7 Normal forces during the transfers



Figure 8 Control torque profiles during the transfers

Allips2 has also been used to estimate the time for liquid motion damp during Sloshsat settling maneuvers attached to the STS. The tank and thruster parameters were given the STS values, and the specified operations scenario was implemented. When STS primary thrust of $7740 \mathrm{~N}$ ( 2 PCRS thrusters) is applied along the tank centerline direction for 20 seconds, it is found that viscous damping parameter $s f$ is to have value 0.05 for the liquid to stop swirling after 5 minutes. The damping of all liquid motion takes considerably longer. If thrust is terminated at the right instance, swirl can be 
absent, or of negligible importance. Figure 9 shows the first 30seconds of the liquid c.o.m trajectory during a 20seconds primary RCS thruster firing. Figure 10 shows the corresponding development of the liquid swirl. Sf value is 0.05 , annotated in the figure. Note that the thrust is cut when the swirl has near-maximum magnitude; the liquid circles the tank in about 4 seconds.

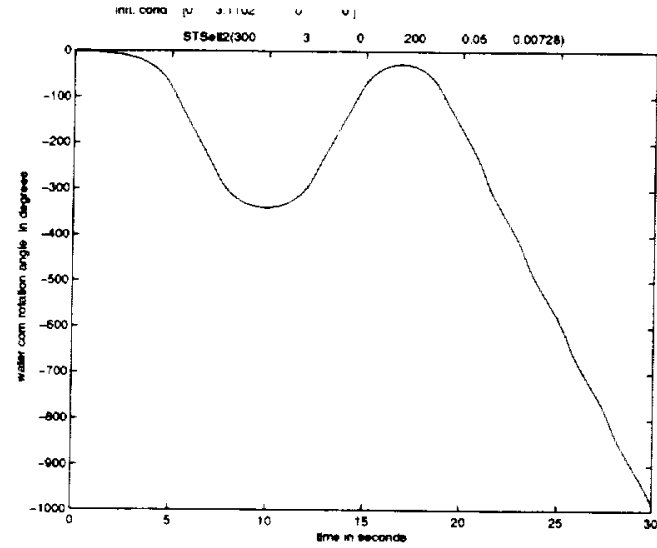

Figure 9 Liquid c.o.m angular location on STS

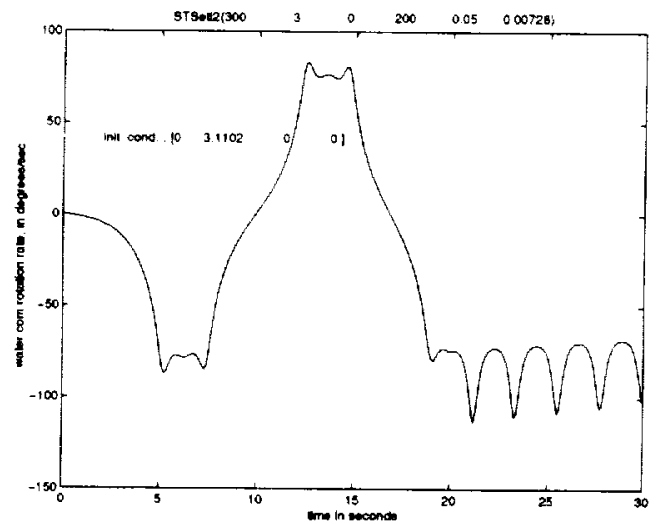

Figure 10 Liquid swirl for 20 seconds STS transfer

\section{VALIDATION OUANTITIES}

The data that are to be produced by Sloshsat are of two types: dynamometric (MSS) data and tank data. MSS stands for Motion Sensing Subsystem, and the data are the output of its six linear accelerometers (Allied Signal QA-3000-010) and three gyroscopes (LITEF $\mu$-FORS). The inertial properties of the empty (dry) Sloshsat are measured accurately which allows to use this invariable rigid body as a sensitive dynamometer for the determination of liquid force and torque. Thruster action is calibrated and so can be accounted for, but should be absent during precise data collection. The tank data are provided by sensors inside the tank such as the Coarse Sensor Array of platinum wire rings discussed earlier.

It is asserted that each of the models discussed before can be validated from MSS data only. This implies that different liquid behaviors will necessarily show up in different force and torque time series. Some candidate validation quantities will be discussed next. If validation cannot be achieved, the tank data will provide the main diagnostic tool. Proper evaluation of the data will require CFD support since the signal is sometimes difficult to interpret. For example, the CSA data between zero and saturation are correlated not only with local liquid height but also with liquid surface inclination and wetted ring fraction.

The models that calculate the tank (spacecraft) dynamics are also able to generate the MSS data and so permit direct comparison between prediction and actual behavior. Integration of these data allow further assessments, e.g. on linear and angular trajectories while events like thruster activation or liquid transit termination will show their signatures. Processing of the data yields the force and torque from the liquid interaction, and with an appropriate model for the liquid dynamics also the liquid c.o.m. trajectory. Evidently, the force and torque must have sufficient magnitude and simulations with SMS indicate that a force value of $0.2 \mathrm{~N}$ might be recoverable. ${ }^{11}$

The reconstitution of the interaction force is of particular interest. Predictions by e.g. SMS or ComFlo show typical spikes in the plot vs. time that can be removed by averaging. In SMS a spike is generated when the slug is at minimum size but still has a velocity component directed to the wall. A collision then reverts this component at a chosen fractional value. Although plausible, such a postulated behavior makes the momentum exchange, and the loss of kinetic energy (damping) dependent on the instantaneous configuration of the liquid and tank c.o.m. locations. ${ }^{14}$ Engel $^{15}$ derives a formula for the loss of kinetic energy following impact of a water drop on a glass wall. This result can be interpreted to give the reconstitution parameter for the collision. Alternative assumptions can be made and may yield the better choice for agreement with spacecraft data. 


\section{SUMMARY}

Sloshsat FLEVO provides an opportunity to obtain experimental results on liquid behavior during maneuvers in the actual low-gravity environment. The motional and tank data allow validation of various models that have been developed for the prediction of these results.

\section{REFERENCES}

1. J.P.B. Vreeburg and David Soo (eds.) Colloquimm on Sloshsat and Liquid Dynamics in Spacecraft. Estec, Noordwijk, NL 16-17 November 1998. ESA WPP 158.

2. David J. Chato. "Planned Axial Reorientation Investigation on Sloshsat." AIAA paper 2000-3441, presented at the $36^{\text {th }}$ Joint Propulsion Conference, Huntsville, AL 16-19 July 2000.

3. J.P.B. Vreeburg. "Dynamics and control of a spacecraft with a moving, pulsating ball in a spherical cavity." Acta Astronautica Vol 40, Nos 2-8, pp. 257274, 1997.

4. D.W. Dunham. "Recovery of NEAR's Mission to Eros." IAF paper 99-Q.5.05, October 1999.

5. H.F. Bauer. "Fluid oscillations in the containers of a space vehicle and their influence upon stability." NASA TR-R-187, February 1964.

6. F.T. Dodge, S.T. Green and M.W. Cruse. "Analysis of small-amplitude low gravity sloshing in axisymmetric tanks." Microgravity Science and Technology Vol IV, No 4, pp. 228-234, 1991.

7. John I. Hochstein, Alfredo E. Patag, and David J. Chato. "Modelling of Impulsive Propellant

Reorientation." NASA TM 101440. January 1989.
8. Douglas B. Kothe, R. C. Mjolsness, and M. D.

Torrey. "RIPPLE: A Computer Program for Incompressible Flows with Free Surfaces" Los Alamos National Laboratory LA-12007-MS, 1991.

9. Jeroen Gerrits and Arthur E.P. Veldman. "Numerical simulation of coupled liquid-solid dynamics." paper to be presented at ECCOMAS 2000, Barcelona, ESP 11-14 September 2000.

10. J.P.B. Vreeburg. "Simulation of liquid dynamics onboard Sloshsat FLEVO." Proceedings STAIF-99, Albuquerque, NM 31 January-2 February 1999, American Institute of Physics CP 458, pp. 836-841.

11. J.P.B. Vreeburg. "Acceleration measurements on Sloshsat FLEVO for liquid force and location determination." Proceedings $4^{\text {th }}$ ESA International Conference on Spacecraft Guidance, Navigation and Control Systems, Estec, Noordwijk, NL 18-21

October 1999, ESA SP-425, pp. 579-585, Feb 2000.

12. Philip G. Good, Anita Carpenter, Howard Flanders and Tom Gardner. "STARDUST TCM and despin in the presence of complex fuel dynamics." AAS paper 98-142.

13. E.W. Otto. "Static and dynamic behavior of the liquid-vapor interface during weightlessness." Aerospace Chemical Engineering, Vol 62, No 61, pp. 158-177, 1964.

14. Rodney Hill. Principles of dynamics. Pergamon. 1964.

15. Olive G. Engel. "Waterdrop Collisions With Solid Surfaces." Journal of Research of the National Bureau of Standards, Vol 54, No 5, pp. 281-298, May 1955. 
Public reporting burden for this collection of information is estimated to average 1 hour per response, including the time for reviewing instructions, searching existing data sources, gathering and maintaining the data needed, and completing and reviewing the collection of information. Send comments regarding this burden estimate or any other aspect of this Davis Highway, Suite 1204. Artington, , and to the Otfice of Management

\begin{tabular}{r|r|r|}
\hline & 3. REPORT TYPE AND DATES COVEAED \\
Technical Memorandum
\end{tabular}

\begin{tabular}{|l|l|l|l|l|}
\hline 4. TITL AND SUBTITE & FUNDING NUMBERS
\end{tabular}

Models for Liquid Impact Onboard Sloshsat FLEVO

6. AUTHOR(S)

WU-242-33-0A-00

Jan P.B. Vreeburg and David J. Chato

7. PERFORMING ORGANIZATION NAME(S) AND ADDRESS(ES)

National Aeronautics and Space Administration

John H. Glenn Research Center at Lewis Field

Cleveland, Ohio 44135-3191

PERFORMING ORGANIZATION REPORT NUMBER

E-12471

9. SPONSORING/MONITORING AGENCY NAME(S) AND ADDRESS(ES)

National Aeronautics and Space Administration

Washington, DC 20546-0001

10. SPONSORINGMONITORING AGENCY REPORT NUMBER

NASA TM-2000-210475

AIAA-2000-5152

\section{SUPPLEMENTARY NOTES}

Prepared for the Space 2000 Conference and Exposition sponsored by the American Institute of Aeronautics and Astronautics, Long Beach, California, September 19-21, 2000. Jan P.B. Vreeburg, National Aerospace Laboratory NLR, Amsterdam, The Netherlands; and David J. Chato, NASA Glenn Research Center. Responsible person, David J. Chato, organization code $5870,216-977-7488$.

12a. DISTRIBUTIONAVAILABILITY STATEMENT

Unclassified - Unlimited

Subject Category: 34

Distribution: Nonstandard

Available electronically at htro://gltrs.grc,nasa,gov/GLTRS

This publication is available from the NASA Center for AeroSpace Information, 301-621-0390.

13. ABSTRACT (Maximum 200 words)

Orbital experiments on the behavior of liquid in spacecraft are planned. The Sloshsat free-flyer is described. Preparation of the experiments, and later evaluation, are supported by models of varying complexity. The characteristics of the models are discussed. Particular attention is given to the momentum transfer between the liquid and the spacecraft, in connection with the liquid impact that may occur at the end of a reorientation maneuver of the spacecraft.

14. SUBJECT TERMS

Fluid dynamics; Spacecraft; Computer modelling

15. NUMBER OF PAGES

13

16. PRICE CODE

17. SECUAITY CLASSIFICATION OF REPORT

Unclassified
18. SECURITY CLASSIFICATION OF THIS PAGE

Unclassified OF ABSTRACT Unclassified 
$-$

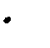


